\title{
Labial fusion in an Adult: A case report
}

\author{
Kesang D Bista, Geeta Gurung, Ashma Rana \\ Dept of Obstetrics \& Gynecology, \\ TUTH, Kathmandu, Nepal
}

\begin{abstract}
:
Labial adhesion in adults is rare and usually due to trauma or chronic inflammation. This is a report of a case of labial fusion in an adult.

A 36 year old unmarried woman presented with the complaint of discomfort during menstruation and difficulty in micturition since one year. The labial adhesion was produced after hot oil application for the treatment of rashes in childhood. Surgical lysis was possible exposing normal genitalia behind the fused labia is reported.
\end{abstract}

Key words: Labial adhesion, labial fusion, micturition difficulty.

\section{Introduction}

Labial fusion is a benign condition defined as partial or complete adhesion of labia minora encountered more in the pediatric population. Although worldwide incidence is not known, in the US it is reported in about $5 \%$ of the pediatric population. Peak incidence is between 12 years. ${ }^{1}$ The etiology is thought to be inflammation / irritation of the labia with denudation of the superficial layer which heals by fibrosis causing adhesions. This inflammation could be due to rash, vulvovaginitis, use of irritants and trauma. Sexual abuse has also been implicated. ${ }^{2}$ Congenital labial fusion can occur in Androgen Insensitivity Syndrome and also following early in utero exposure to androgens or danazol. Most of the literature only refers to this condition in children. its occurrence in adults being very uncommon. This is probably due to the fact that spontaneous resolution usually occurs or treatment with either estrogen creams or lysis has been done early. ${ }^{33}$

\section{Case report}

A 36 year old lady from Kathmandu was admitted from the OPD the complaint of difficulty in micturition for the last a year, which occurred only during menstruation. There was no history of burning, micturition frequency or dribbling of the urine during menses but there was certain discomfort and uneasiness and a feeling of heaviness (retention of clotted blood) in the vagina as if there was insufficient passage for the free passage of the menstrual blood giving a sense of heaviness at the introitus. Her menses till date had been regular with average flow with no dysmenorrhea.
She gave a history of some rash in infancy for which hot oil was applied by her mother. She was unmarried and this was the first time she had sought any medical opinion General and systemic examinations were normal, with normal secondary sexual features. Per vaginal examination revealed a normal mons and hair distribution, but the whole introitus was covered by a thick band spreading between the labia majora. Two small openings were present, one at the upper end and the other one in the middle of the adhesion, through which a rubber catheter could be passed and connected. Ultrasonography revealed a normal uterus and adnexae except for a small right kidney. Intravenous urography showed normal urinary system. After proper counseling and ensuring surgical fitness, lysis was performed under general anesthesia initiated from the lower opening with guidance by a metal catheter passed through the upper opening (Fig1). Cutting away the adhesion revealed a normal nulliparous cervix, vagina and normal external genitalia. Excess tissue was trimmed off and the remaining cut margin sutured with catgut (Fig 2).The post operative period was uneventful and the patient was discharged on the $5^{\text {th }}$ post operative day with advice for follow up and the possibility of a normal sexual life.

\section{Comment}

This case demonstrates that not all labial fusion encountered in early life resolve in later years. In this case, lack of knowledge that correction was possible led to late detection. . There are few other reports in the literature of labial adhesions in pubertal girls as a consequence of infection like lichen sclerosis or trauma 


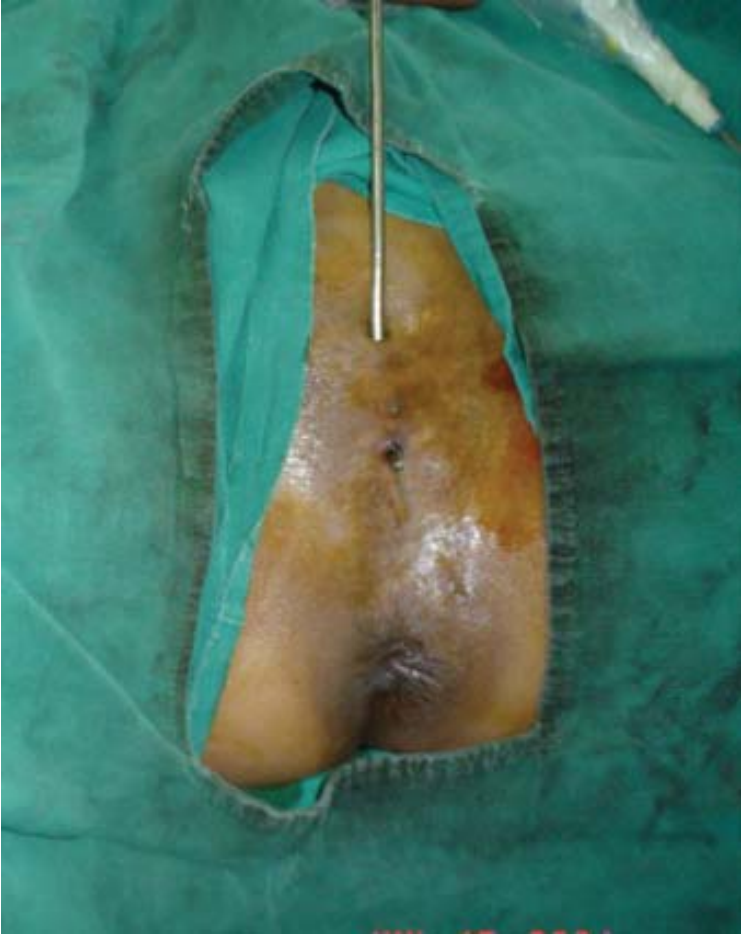

Figure 1 metal catheter being passed from upper opening

acquired spontaneously or following circumcision and sexual abuse whereas

In adults it could also follow complications of surgery like pelvic floor repair or rarely following delivery. ${ }^{4-6}$

In the event of early treatment, follow up is needed to ensure that spontaneous lysis has occurred or in failure to do so, a definitive surgical intervention is needed. This patient probably would have come earlier if she had been married but as she remained unmarried she only came due to her menstrual problem. In this case the small opening in the middle of the adhesion probably facilitated the drainage of urine and menstrual blood (fig1). Otherwise most children present with urinary dribbling on standing or repeated urinary tract infection that may help in making diagnosis during childhood. Although the first mode of treatment is estrogen cream, medical management does not suffice in long standing cases as the adhesion becomes thicker and fibrosed and surgical lysis is necessitated. Amniotic membrane grafting has also been mentioned in some refractory cases.

\section{Conclusion}

Labial fusion is uncommon in adults. The prognosis is good with surgical lysis which yields good results.

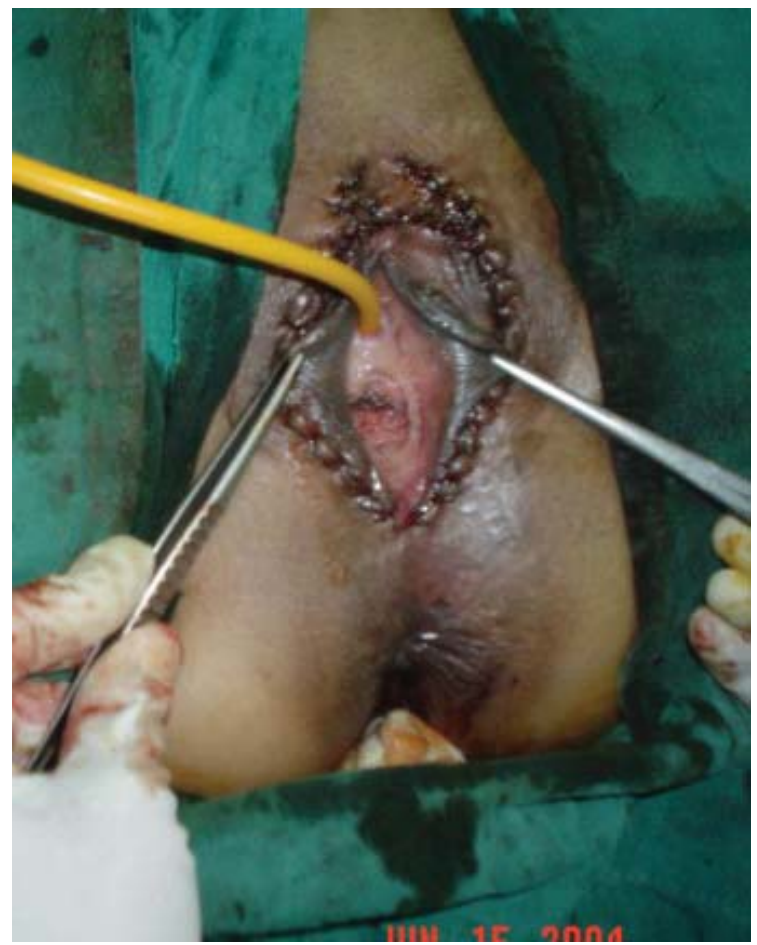

Figure 2 the final look.

Early diagnosis and intervention could have prevented the morbidity that she faced prior to diagnosis.

\section{References}

1. Leung AK, Robson W L and Tay Uyboco J. The incidence of labial fusion in children. J Pediatr Child Health 1993; 29: 235-236.

2. Kellog ND, Para JM and Menard S. Children with anogenital symptoms and signs referred for sexual abuse evaluations. Arch Pediatr Adolesc Med 1998; 152: 634-641.

3. Leung AK, RobsonWL, Kao CP et al. Treatment of labial fusion with topical estrogen therapy. Clin Pediatr 2005; 44: 245-247.

4. Awang N .Report of labial fusion in pubertal girl with urinary signs and symptoms-a delayed consequence of female circumcisions. The Aust NZ J Obstet Gynecol 2004; 44: 4.

5. KumarKR, Aggarwal S, Chanana C, Kumar S and Malhotra N. Arch Obstet Gynecol 2006;(273) : 243-245.

6. Sharma B, Arora R and Preston J. Postpartum labial adhesions following normal vaginal delivery. J Obstet Gynecol 2005; 25: 215.

7. Amniotic membrane grafting to treat refractory labial adhesions postpartum. A case report J Reprod Med 2002; 47: 235-237. 